\title{
Reconciling quantum trajectories and stationary quantum distributions in single-photon polarization states
}

\author{
Alfredo Luis ${ }^{1}$ and Ángel S. Sanz $z^{2,3}$ \\ ${ }^{1}$ Departamento de Óptica, Facultad de Ciencias Físicas, Universidad Complutense, 28040 Madrid, Spain \\ ${ }^{2}$ Instituto de Física Fundamental (IFF-CSIC), Serrano 123, 28006 Madrid, Spain \\ ${ }^{3}$ Department of Physics and Astronomy, University College London, Gower Street, London WC1E 6BT, United Kingdom
}

(Received 10 February 2013; published 27 June 2013)

\begin{abstract}
The question of the representation of quantum stationary partially polarized waves as random superpositions of different polarization ellipses is addressed. To this end, the Bohmian formulation of quantum mechanics is considered and extended to quantum optical polarization. As is shown, this approach properly combines definite time-evolving trajectories with rigorous stationary quantum distributions via the topology displayed by the associated phase field.
\end{abstract}

DOI: 10.1103/PhysRevA.87.063844

PACS number(s): 42.50.Ct, 42.25.Ja, 03.50.De, 03.65.Ta

\section{INTRODUCTION}

In most courses, textbooks, and specialized treatises it is common to introduce the polarization of harmonic waves as an ellipse described by the electric field in a real configuration representation [1]. Within this approach, partial polarization thus arises by the rapid and random succession of more or less different polarization states. In more advanced approaches, though, the Stokes parameters and Poincaré sphere are introduced, which allow us to assess the degree of polarization and make use of the powerful tools provided by the SU(2) group. Moreover, the Stokes parameters also enable a simple connection to the quantum regime in terms of their quantum counterparts, namely, the Stokes operators [2].

Once the Stokes parameters are introduced, usually the polarization ellipses are left behind, so that the statistics of the electric field in the polarization plane are abandoned. This is particularly remarkable within the quantum domain, where uncertainty relations imply that no field state can describe a perfect ellipse, just in the same way that no particle can follow a definite trajectory [3-5]. This thus suggests some kind of quantum mismatching between the intuitive ellipse picture and the more powerful Stokes-Poincare formalism. More specifically, we find quantum states with the maximum degree of polarization, but with their electric-field distribution far from resembling any ellipse, as seen, for example, in Fig. 2 below (for other examples, see also Fig. 1 in Ref. [3] or Fig. 4 in Ref. [4]). Furthermore, the mismatch seems aggravated for quantum stationary states (i.e., states with definite total photon number). In such a case, the electric-field distribution is constant even for states with the maximum degree of polarization, which should correspond to an electric field rapidly describing an elliptical trajectory.

In this work we investigate whether there is still a possibility to keep the most intuitive approach, where partially polarized waves are devised as random superpositions of different polarization ellipses. To this end, the Bohmian formulation of quantum mechanics [6] or, more properly speaking, its extension to optics $[7,8]$, seems to be very convenient. This approach properly combines the two ideas that we want to combine: definite trajectories and rigorous quantum distributions. More specifically, here we are going to study the Bohmian trajectories (or optical paths) for a two-dimensional (2D) isotropic harmonic oscillator, which properly represents in the quantum domain a transversal two-mode harmonic wave. For definiteness, and for the sake of simple illustration, we consider single-photon pure states. Accordingly, we have organized this work as follows. In Sec. II we introduce the basic elements involved in the representation of single-photon polarization states. In Sec. III the associated Bohmian dynamics is analyzed and discussed. Finally, in Sec. IV the main conclusions drawn from this work are summarized.

\section{POLARIZATION OF ONE-PHOTON STATES}

\section{A. Polarization ellipse}

Consider a harmonic light wave consisting of two modes of the same frequency $\omega$, and with their corresponding electric fields vibrating along orthogonal directions. These two modes are represented by their complex amplitude operators $\hat{a}_{1}$ and $\hat{a}_{2}$. This system is equivalent to a particle in a $2 \mathrm{D}$ isotropic harmonic potential. The equivalence becomes clearer through the quadrature operators representing the real and imaginary parts of the electric field, $\hat{a}_{j} \propto \hat{X}_{j}+i \hat{Y}_{j}$, with

$$
\hat{X}_{j}=\frac{1}{\sqrt{2}}\left(\hat{a}_{j}^{\dagger}+\hat{a}_{j}\right), \quad \hat{Y}_{j}=\frac{i}{\sqrt{2}}\left(\hat{a}_{j}^{\dagger}-\hat{a}_{j}\right),
$$

and commutator $\left[\hat{X}_{j}, \hat{Y}_{k}\right]=i \delta_{j k}$, with $j, k=1,2$. The $\hat{X}_{j}$ and $\hat{Y}_{j}$ operators are thus formally equivalent, respectively, to the (dimensionless) position and momentum of a $2 \mathrm{D}$ massive particle. For simplicity, and to exploit as much as possible this equivalence, we assume $\hbar=1, \omega=1$, and $m=1$, with the latter being the mass of the equivalent effective particle. The corresponding effective Hamiltonian then reads as $\hat{\mathcal{H}}=$ $\hat{a}_{1}^{\dagger} \hat{a}_{1}+\hat{a}_{2}^{\dagger} \hat{a}_{2}$.

The quadrature operators $\hat{X}_{j}$ (with $j=1,2$ ) also allow us to introduce a wave function for the $2 \mathrm{D}$ real electric field by projection of the field state $|\psi\rangle$ on the unnormalized joint eigenstate of the quadrature operators $\hat{X}_{j}\left|x_{1}, x_{2}\right\rangle=$ $x_{j}\left|x_{1}, x_{2}\right\rangle$. In particular, for photon-number eigenstates, with $\hat{a}_{j}^{\dagger} \hat{a}_{j}\left|n_{1}, n_{2}\right\rangle=n_{j}\left|n_{1}, n_{2}\right\rangle$, we have

$$
\left\langle x_{1}, x_{2} \mid n_{1}, n_{2}\right\rangle=\mathcal{N}_{12} H_{n_{1}}\left(x_{1}\right) H_{n_{2}}\left(x_{2}\right) e^{-\left(x_{1}^{2}+x_{2}^{2}\right) / 2},
$$


where $\mathcal{N}_{12}=\left(2^{n_{1}+n_{2}} \pi n_{1} ! n_{2} !\right)^{-1 / 2}$ is the norm and $H_{n_{j}}$ are the corresponding Hermite polynomials. Accordingly, the quantum analog of the polarization ellipse is the distribution for the 2D real electric field [3-5],

$$
P\left(x_{1}, x_{2}, t\right)=\left|\left\langle x_{1}, x_{2} \mid \psi(t)\right\rangle\right|^{2},
$$

with $|\psi(t)\rangle=\exp (-i \hat{\mathcal{H}} t)|\psi(0)\rangle$.

\section{B. Stokes parameters}

Regarding the Stokes picture of polarization, it can be well started from the Stokes operators [2]

$$
\begin{array}{ll}
\hat{S}_{0}=\hat{a}_{1}^{\dagger} \hat{a}_{1}+\hat{a}_{2}^{\dagger} \hat{a}_{2}, & \hat{S}_{x}=\hat{a}_{1}^{\dagger} \hat{a}_{1}-\hat{a}_{2}^{\dagger} \hat{a}_{2}, \\
\hat{S}_{y}=\hat{a}_{2}^{\dagger} \hat{a}_{1}+\hat{a}_{2} \hat{a}_{1}^{\dagger}, \quad \hat{S}_{z}=i\left(\hat{a}_{2} \hat{a}_{1}^{\dagger}-\hat{a}_{2}^{\dagger} \hat{a}_{1}\right),
\end{array}
$$

which satisfy the commutation relations

$$
\left[\hat{S}_{x}, \hat{S}_{y}\right]=-2 i \hat{S}_{z}
$$

with cyclic permutations, and

$$
\hat{\mathbf{S}}^{2}=\hat{S}_{0}\left(\hat{S}_{0}+2 \hat{\mathbb{I}}\right), \quad\left[\hat{\mathbf{S}}, \hat{S}_{0}\right]=\hat{\mathbf{0}}
$$

with $\hat{\mathbf{S}}=\left(\hat{S}_{x}, \hat{S}_{y}, \hat{S}_{z}\right)$. The classical Stokes parameters are the mean values of the Stokes operators, $s_{j}=\left\langle\hat{S}_{j}\right\rangle$. Because of the nonvanishing commutator, as described by Eq. (5), no state can have definite values of all Stokes operators simultaneously (the only exception being the two-mode vacuum, where they vanish trivially). This is conveniently expressed by the uncertainty relation

$$
\begin{aligned}
(\Delta \hat{\mathbf{S}})^{2} & =\left(\Delta \hat{S}_{x}\right)^{2}+\left(\Delta \hat{S}_{y}\right)^{2}+\left(\Delta \hat{S}_{z}\right)^{2} \\
& =\left\langle\hat{S}_{0}\left(\hat{S}_{0}+2 \hat{\mathbb{I}}\right)\right\rangle-\langle\hat{\mathbf{S}}\rangle^{2} \geqslant 2\left\langle\hat{S}_{0}\right\rangle,
\end{aligned}
$$

which holds after Eq. (6) [9], taking into account that $|\langle\hat{\mathbf{S}}\rangle| \leqslant\left\langle\hat{S}_{0}\right\rangle$.

Consider now the standard (classical) definition of the degree of polarization $\mathcal{P}=|\boldsymbol{s}| / s_{0}$. Some other more complete definitions, though, have also been proposed, particularly within the quantum domain $[4,5,10]$. Based on the fact that $|s| \leqslant s_{0}$, the Stokes parameters readily provide a representation for polarization states in a unit sphere, namely, the Poincaré sphere. This is done through the parametrization

$$
\begin{aligned}
& s_{x}=s_{0} \cos (2 \chi) \cos (2 \varphi), \quad s_{y}=s_{0} \cos (2 \chi) \sin (2 \varphi), \\
& s_{z}=s_{0} \sin (2 \chi),
\end{aligned}
$$

which is sketched in Fig. 1. In the transformation relations (8), $\tan (2 \varphi)=s_{y} / s_{x}$, with $\varphi$ being the angle between the major axis of the polarization ellipse and the $x_{1}$ axis. Regarding $\chi$, this angle determines the ratio between the minor and major axes of the polarization ellipse ( $b$ and $a$, respectively), with $\pi / 4 \geqslant \chi \geqslant-\pi / 4$ and $\tan \chi= \pm b / a$, where the sign is given by the handedness (right handed if $s_{z}>0$ and left handed if $s_{z}<0$ ). The relations (8) thus provide us with a one-to-one correspondence between the Stokes parameters (or points on the Poincaré sphere) and some average or mean polarization ellipse.
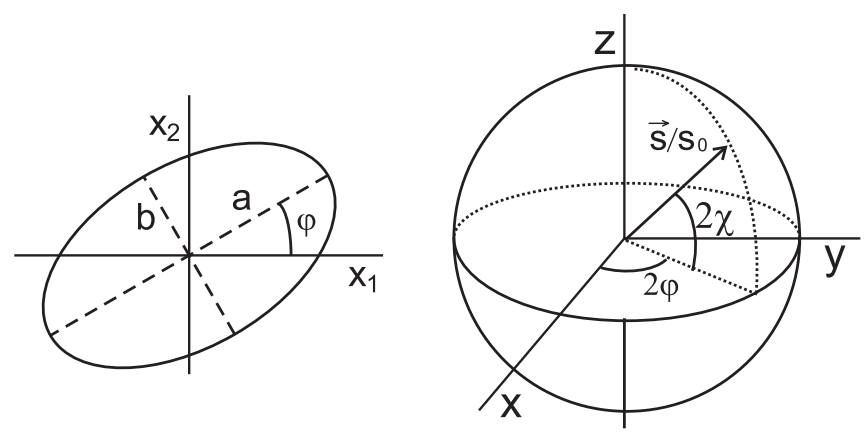

FIG. 1. Polarization ellipse (left) and Poincaré sphere (right).

\section{One-photon states}

Let us now consider, more specifically, the most general pure one-photon state. In the photon-number basis $\left|n_{1}, n_{2}\right\rangle$ it reads as

$$
|\psi\rangle=c_{1}|1,0\rangle+c_{2}|0,1\rangle,
$$

with Stokes parameters

$$
\begin{aligned}
& s_{0}=\left|c_{1}\right|^{2}+\left|c_{2}\right|^{2}=1, \quad s_{x}=\left|c_{1}\right|^{2}-\left|c_{2}\right|^{2}, \\
& s_{y}=c_{1}^{*} c_{2}+c_{1} c_{2}^{*}=2 \operatorname{Re}\left(c_{1} c_{2}^{*}\right), \\
& s_{z}=i\left(c_{1}^{*} c_{2}-c_{1} c_{2}^{*}\right)=2 \operatorname{Im}\left(c_{1} c_{2}^{*}\right) .
\end{aligned}
$$

These are all stationary states, i.e., $\hat{\mathcal{H}}|\psi\rangle=|\psi\rangle$, so that $|\psi(t)\rangle=\exp (-i t)|\psi(0)\rangle$. They are also SU(2) coherent states [11], which are usually considered as classical-like regarding polarization [12], as well as being minimum-uncertainty states of the uncertainty relation (7). Moreover, since $|\boldsymbol{s}|=s_{0}$ for these states, we have $\mathcal{P}=1$, thus displaying the maximum degree of polarization according to the classic definition seen above. In spite of this, they cannot be considered as having perfect polarization, since $(\Delta \hat{\mathbf{S}})^{2}=2\left\langle\hat{S}_{0}\right\rangle \neq 0$. This is properly reflected by more complete assessments of the degree of polarization $[4,5,10]$.

The quadrature wave function associated with the pure onephoton state (9) is

$$
\left\langle x_{1}, x_{2} \mid \psi(t)\right\rangle=\sqrt{\frac{2}{\pi}}\left(c_{1} x_{1}+c_{2} x_{2}\right) e^{-\left(x_{1}^{2}+x_{2}^{2}\right) / 2-i t},
$$

and the corresponding electric-field distribution is

$$
P\left(x_{1}, x_{2}\right)=\frac{1}{\pi}\left[\left(1+s_{x}\right) x_{1}^{2}+\left(1-s_{x}\right) x_{2}^{2}+2 s_{y} x_{1} x_{2}\right] e^{-\left(x_{1}^{2}+x_{2}^{2}\right)},
$$

which is effectively independent of time, i.e., it is stationary, as expected. In Fig. 2 we show a series of contour plots of $P\left(x_{1}, x_{2}\right)$ for angles $2 \chi$ and $2 \varphi$ that cover the first quadrant of the Poincare sphere; the behavior in the remaining quadrants is analogous and can be inferred by taking into account the corresponding symmetry relations. Also, for convenience regarding the computation of the Bohmian trajectories (see the discussion below), we have considered the minimum $2 \chi$ to be $\pi / 10$ instead of 0 . As can be seen, only for $2 \chi= \pm \pi / 2$ and any $\varphi$ does the picture of an ellipse remain valid (this is for circularly polarized light). As the value of these angles is changed, this picture breaks down despite having the maximum degree of polarization in any of them $(\mathcal{P}=1)$, 

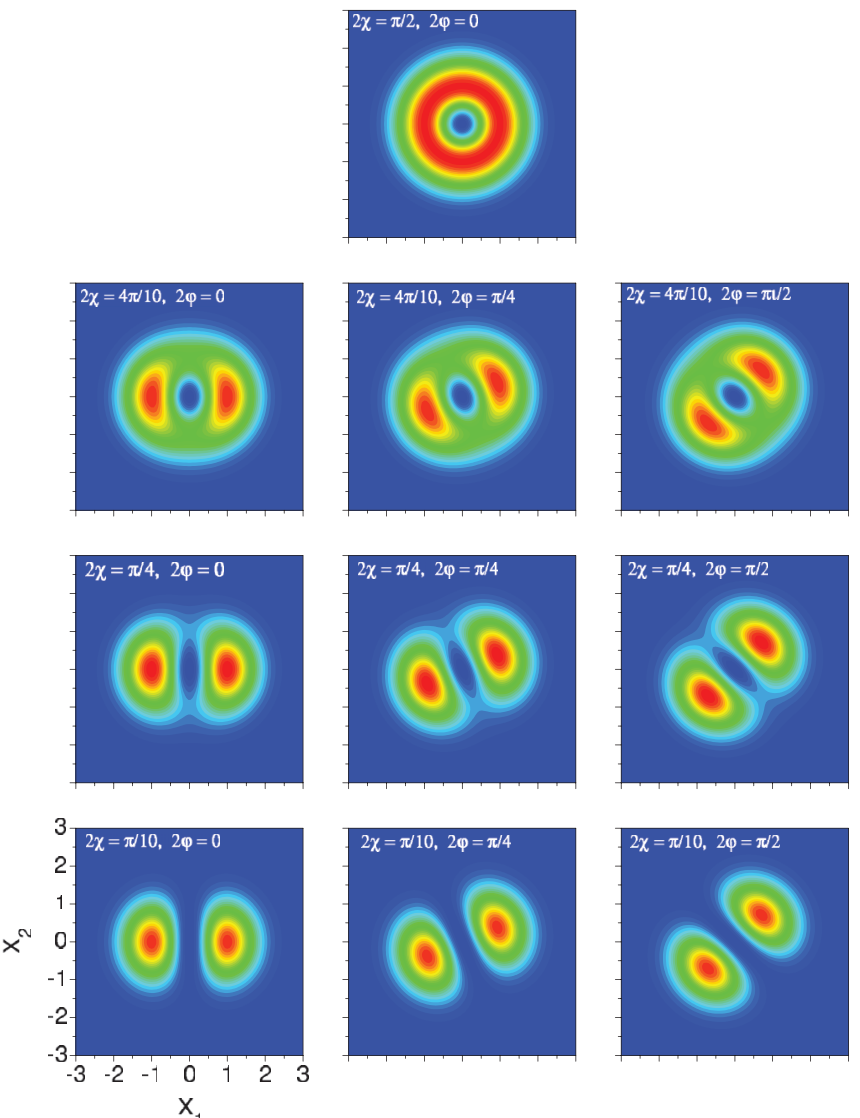

FIG. 2. (Color online) Contour plots corresponding to the $P\left(x_{1}, x_{2}\right)$ distribution of the 2D real electric field associated with the pure one-photon state (9). The values chosen for the angles $2 \chi$ and $2 \varphi$ cover the first quadrant of the Poincaré sphere (see Fig. 1, right). Minimum to maximum is indicated with the transition from blue to red, starting from zero in all cases.

with the most dramatic breaking off being for $2 \chi=0$, i.e., along the equator of the sphere (this is for linearly polarized light). In this case, $P\left(x_{1}, x_{2}\right)$ essentially consists of two separate lobes. For $2 \varphi=0$ these lobes are parallel to the $x_{1}$ axis (see the lowest left panel), but their inclination changes as $2 \varphi$ increases, thus becoming parallel to the $x_{2}$ axis when $2 \varphi=\pi$ (for the intermediate case, $2 \varphi=\pi / 2$, they are $\pi / 4$ inclined, as seen in the lowest right panel). Apart from deviating from the ellipsoidal picture, it is also worth stressing that information about the handedness will also get lost. This is because $P\left(x_{1}, x_{2}\right)$ does not depend on $s_{z}$.

\section{BOHMIAN DYNAMICS}

\section{A. Equations of motion}

Let us now consider the Bohmian approach with the purpose of grasping some physical insight from the dynamical state associated with the wave function (11). Within this context, the corresponding Bohmian trajectories $\boldsymbol{r}(t)=\left[x_{1}(t), x_{2}(t)\right]$ are obtained after integration of the guidance equation

$$
\dot{\boldsymbol{r}}=\nabla S=-i e^{-i S} \nabla e^{i S},
$$

where $S$ is the (real-valued) phase of the wave function (11), i.e.,

$$
e^{i S}=\frac{\left(c_{1} x_{1}+c_{2} x_{2}\right) e^{-i t}}{\sqrt{\left|c_{1}\right|^{2} x_{1}^{2}+\left|c_{2}\right|^{2} x_{2}^{2}+\left(c_{1} c_{2}^{*}+c_{1}^{*} c_{2}\right) x_{1} x_{2}}} .
$$

In terms of the Stokes parameters, this expression can also be recast as

$$
e^{i S}=\frac{\sqrt{1+s_{x}}\left[x_{1}+\left(\frac{1-s_{x}}{s_{y}+i s_{z}}\right) x_{2}\right] e^{-i t+i \delta}}{\sqrt{x_{1}^{2}+x_{2}^{2}+s_{x}\left(x_{1}^{2}-x_{2}^{2}\right)+2 s_{y} x_{1} x_{2}}},
$$

where $\delta$ is a global relative phase associated with the coefficient $c_{1}$. This phase factor is physically meaningless regarding the topology displayed by the phase field, $S$, as well as the trajectory dynamics described by the equation of motion below, as also happens with $t$. Substituting this expression into Eq. (13), we find

$$
\dot{\boldsymbol{r}}=\frac{s_{z}}{x_{1}^{2}+x_{2}^{2}+s_{x}\left(x_{1}^{2}-x_{2}^{2}\right)+2 s_{y} x_{1} x_{2}}\left(x_{2},-x_{1}\right) .
$$

In general, Eq. (16) has to be numerically integrated in order to obtain the corresponding trajectories, as seen in Sec. III C below. However, as shown in the next section, it is also possible to draw a series of interesting conclusions directly from the form of Eq. (16), without the need to integrate it.

\section{B. Properties of the trajectories}

\section{Circular trajectories}

In spite of the complex dependence on $x_{1}$ and $x_{2}$ displayed by the prefactor of the equation of motion (16), the trajectories for any single-photon state with $s_{z} \neq 0$ are always circular. This comes from the fact that

$$
\boldsymbol{r} \cdot \nabla S=\boldsymbol{r} \cdot \dot{\boldsymbol{r}}=0,
$$

which implies that $\boldsymbol{r}^{2}$ (and therefore $\boldsymbol{r}$ ) is constant.

Now, for $s_{z}=0$, when the photons are linearly polarized, we have $\dot{\mathbf{r}} \equiv \mathbf{0}$ and therefore the whole vector $\boldsymbol{r}$ will be constant. That is, each separate $\mathbf{r}$ component is constant with time, since Eq. (17) ensures the time independence only of $r=|\mathbf{r}|$, but not its components (see below). This case can be then regarded as the limit of a circle described at a vanishing speed.

\section{Nonuniform angular frequency}

The angular frequency of these circular trajectories is not uniform. This is readily seen if we use polar coordinates,

$$
x_{1}=r \cos \phi, \quad x_{2}=r \sin \phi,
$$

and then express (16) in terms of these coordinates, which renders the following equation of motion for the polar component:

$$
\begin{aligned}
\dot{\phi} & =-\frac{s_{z} / r^{2}}{1+s_{x} \cos 2 \phi+s_{y} \sin 2 \phi} \\
& =-\frac{\sin (2 \chi) / r^{2}}{1+\cos (2 \chi) \cos (2 \phi-2 \varphi)} .
\end{aligned}
$$

We recall that the polarization ellipse is described at constant angular speed $\omega$ (in our case $\omega=1$ ). However, as seen through 
(19), this is not the case for the Bohmian trajectories, where $\dot{\phi}$ strongly depends on the polarization state (see below). Thus, the only case with uniform angular velocity holds for $\chi=$ $\pm \pi / 4$, i.e., for circularly polarized light.

\section{Handedness}

The handedness of the Bohmian trajectories is the same as for the corresponding polarization ellipses. After the change of coordinates (18), the Bohmian motion will be right handed if $\dot{\phi}<0$, while the polarization ellipse is right handed when $s_{z}>0$.

\section{Extreme instantaneous frequencies and ellipse parameters}

The maximum instantaneous angular frequency of the Bohmian trajectories, denoted by $\Omega=|\dot{\phi}|$, is

$$
\Omega_{\max }=\frac{1}{r^{2}|\tan \chi|},
$$

which holds when $\cos (2 \phi-2 \varphi)=-1$, i.e., when $\phi=\varphi \pm$ $\pi / 2$, modulo $\pi$. In other words, this condition is equivalent to saying that the maximum instantaneous angular frequency of the Bohmian trajectory coincides with the direction of the minor axis of the polarization ellipse (see Fig. 1).

On the contrary, the minimum instantaneous angular frequency is

$$
\Omega_{\min }=\frac{|\tan \chi|}{r^{2}}
$$

which holds when $\cos (2 \phi-2 \varphi)=1$, i.e., when $\phi=\varphi$, modulo $\pi$. That is, the minimum instantaneous angular frequency coincides with the direction of the major axis of the polarization ellipse. Note, therefore, that the ratio between minimum and maximum instantaneous angular frequencies is

$$
\frac{\Omega_{\min }}{\Omega_{\max }}=\tan ^{2} \chi=\frac{b^{2}}{a^{2}},
$$

which coincides with the ratio of the minimum to maximum axes of the polarization ellipse, $b$ and $a$, respectively.

\section{Compatibility with classical electrodynamics and nonclassicality}

We recall that the variables $x_{1}$ and $x_{2}$ are two orthogonal electric-field components, so that the associated $x_{1}(t)$ and $x_{2}(t)$ Bohmian trajectories represent the dynamical evolution of the electric field. These orbits are not compatible with the Maxwell equations, which demand that the electric field describes an ellipse at constant angular frequency $\omega$ (in our case $\omega=1$ ). The discrepancy between Bohmian orbits and classical electromagnetism is apparent through Eq. (16). This equation, which expresses the time derivative of the electric field vector, is strongly nonlinear. This is also clearly displayed in Figs. 3, 4, and 5 below, where the jumping behavior of the angular frequencies $\dot{\phi}$ are irreconcilable with the harmonic behavior of classical polarization ellipses.

We find this lack of compatibility with classical electrodynamics very suggestive, since it may be naturally ascribed to the nonclassical nature of stationary one-photon field states (9). Of course, it can be readily shown that for nonstationary, classical-like Glauber coherent states the Bohmian orbits are
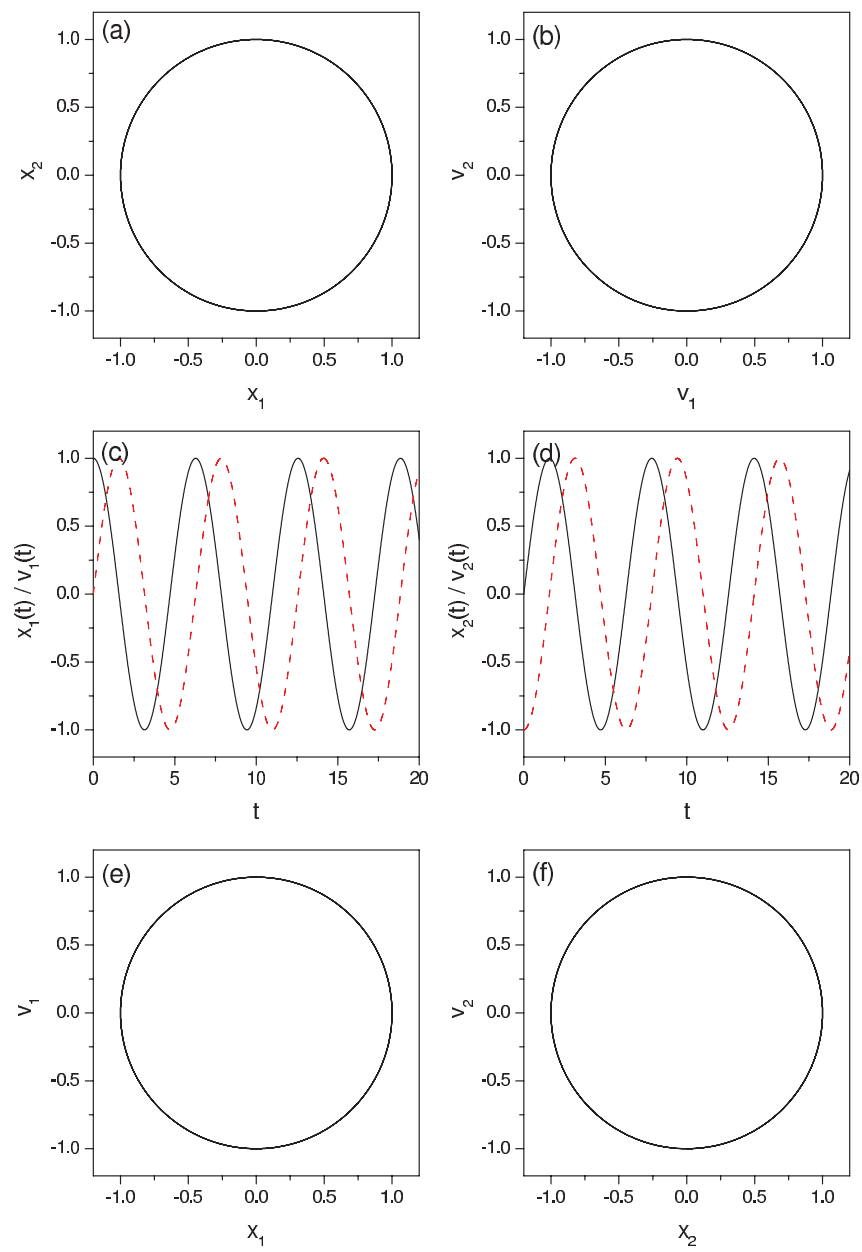

FIG. 3. (Color online) Different representations of the Bohmian trajectories associated with a single-photon polarization state characterized by $2 \chi=\pi / 2$ and $2 \varphi=0$ on the Poincaré sphere. In (c) and (d) the black solid lines denote the spatial component ( $x_{1}$ or $\left.x_{2}\right)$, while the red dashed line refers to the respective velocity component $\left(v_{1}\right.$ or $v_{2}$ ). In the corresponding panels, the velocity components have been obtained by evaluating (16) along the trajectory.

actually ellipses described at constant angular frequency $\omega$, in full agreement with classical optics. However, the mechanism that generates the dynamics in this case is very different from the one involved in stationary single-photon states. In the case of coherent states the dynamics appears because the phase of the corresponding wave function is time dependent, while in the latter it is just a purely topological property associated with phase-local (time-independent) space variations. In this regard, notice that the rich and nontrivial dynamics displayed by the one-photon orbits is needed to reconcile electric-field dynamics with the counterintuitive stationary electric-field probability distribution associated with nonclassical photonnumber light states. This may provide a different perspective on the quantum nature of light states.

\section{Numerical simulations}

A priori, the properties discussed above may seem counterintuitive and puzzling. For example, how is it possible that the trajectories describe circles if their angular frequency is 

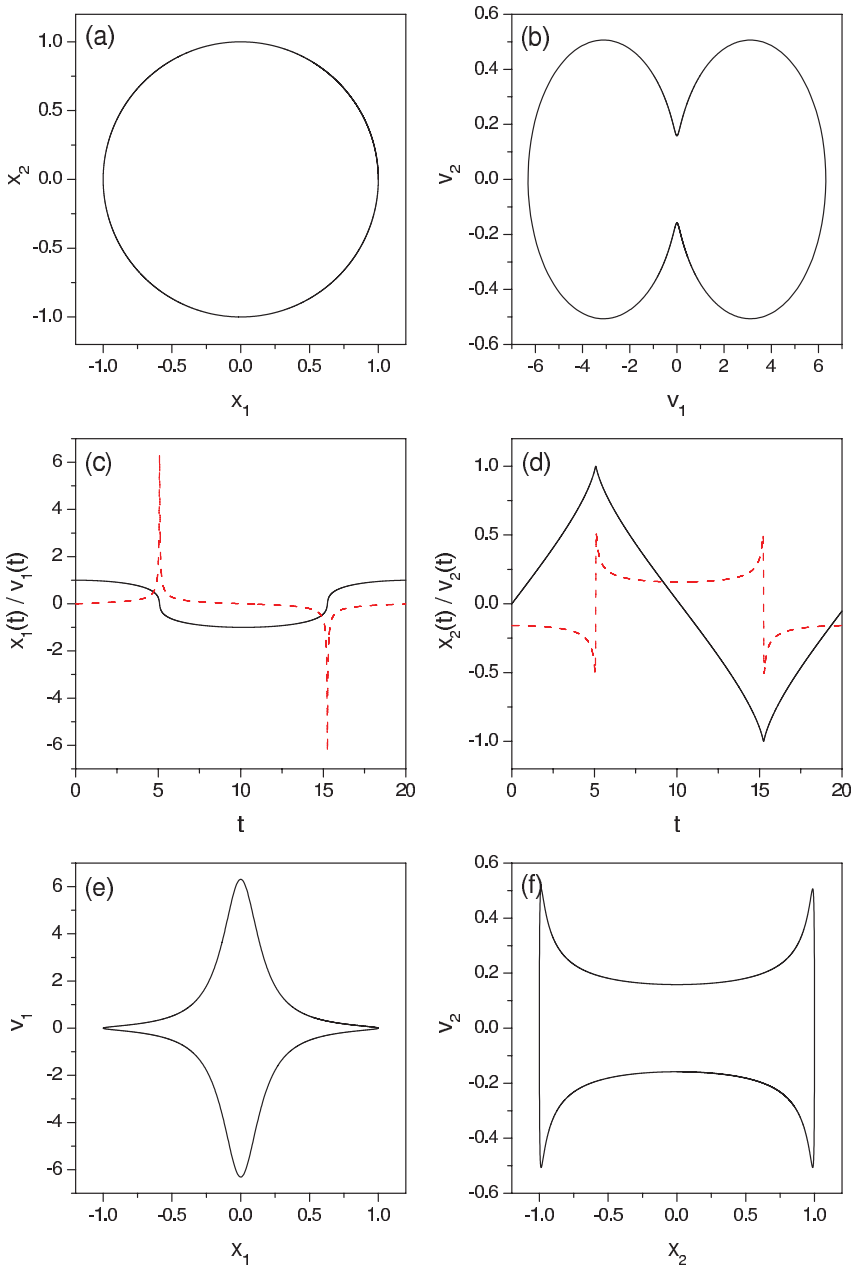

FIG. 4. (Color online) As in Fig. 3, but for $2 \chi=\pi / 10$ and $2 \varphi=0$.

variable with time, or if they tend to distribute in regions where $P\left(x_{1}, x_{2}\right)$ is maximum, avoiding the nodal ones? Actually, it seems that there is a mismatching between the symmetry of the wave function and the features characterizing the trajectories. In order to elucidate these questions as well as to reconcile two antagonists, namely, motion and stationarity in the case of single-photon states accounted for by wave functions like (9), we have numerically integrated the equation of motion (16) for some of the conditions considered in Fig. 2. Each condition represents a different single-photon state vector and, therefore, a particular dynamics. The corresponding trajectories as well as some other additional representations of interest are displayed in Figs. 3, 4, and 5. For clarity, the initial condition, $x_{1}(0)=1$ and $x_{2}(0)=0$, was chose to be the same in the three cases. Accordingly, all the trajectories will be circles of unit radius and, in principle, indistinguishable, in agreement with (17). Disambiguation follows, though, when one analyzes the time dependence of each component separately or their respective velocities, as can be seen in the additional representations (see also the discussions below). For completeness, and also to verify our conclusions in other cases, we also considered the same cases, but with other radii. The corresponding trajectories will not be plotted here, although we would like to stress the fact that we could corroborate that, effectively, the smaller the radius, the faster the motion
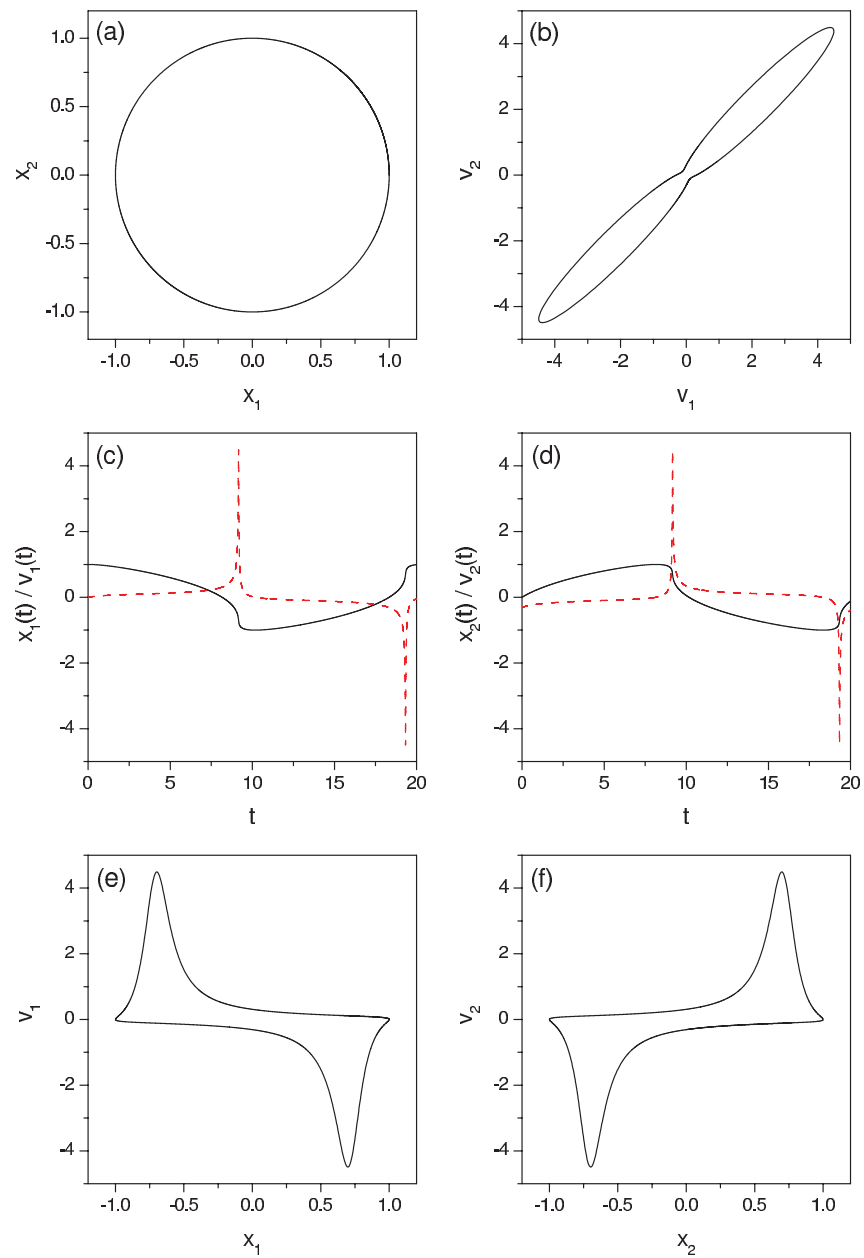

FIG. 5. (Color online) As in Fig. 3, but for $2 \chi=\pi / 10$ and $2 \varphi=\pi / 2$.

around the circle, in agreement with the $r^{-2}$ factor in Eq. (19). Moreover, it has also been noticed that the time needed for trajectories with the same radius to complete the circle is different, increasing as $2 \chi$ approaches zero. This can be seen below by examining the dependence on time of the velocity components of the trajectories, i.e., $v_{i}=\dot{x}_{i}$, with $i=1,2$ [see the red dashed line in panels (c) and (d) of Figs. 3, 4, and 5]. Thus, as $2 \chi$ approaches zero, one observes a certain bistable behavior: $v_{1}$ and $v_{2}$ undergo important variations in extremely short periods of time, while they remain finite (or even vanishing) for relatively long times, thus conferring a certain stationarity or stability to the corresponding photon state. This fact is actually in correspondence with the last of the properties studied in Sec. III B.

Going now to each particular case, in Fig. 3 we have displayed the results for circularly polarized light $2 \chi=\pi / 2$ and $2 \varphi=0$ on the Poincaré sphere (see Fig. 1). Notice that the evolution is ruled by a harmonic motion. This can be seen through the time dependence of the components $x_{1}$ and $x_{2}$, and their corresponding velocities $v_{1}$ and $v_{2}$ [see Figs. 3(c) and 3(d)], or through the respective phase-space orbits [see Figs. 3(e) and 3(f)]. In this case, $s_{z}=s_{0}=1$ and $s_{x}=s_{y}=0$, which means that the photon has equal probability to be in the $|1,0\rangle$ state as in the $|0,1\rangle$ state. Therefore, this uncertainty leads the trajectory 
to visit all points on a circle of a given radius at the same (angular) velocity, this eventually manifesting as a harmoniclike motion or oscillation between $|1,0\rangle$ and $|0,1\rangle$ (or, in other terms, between the horizontal and vertical polarization states).

As we move towards the equator of the Poincaré sphere, i.e., vanishing $s_{z}$ for linearly polarized light (from top to bottom in Fig. 2), the toruslike distribution starts developing two lobes, which in the limit $s_{z}=0$ become separate. Here we find an apparently paradoxical behavior: $P\left(x_{1}, x_{2}\right)$ consists of two separate lobes, but the trajectories are still circles, as indicated by (17). To analyze this situation, we have proceeded as before, but considering the single-photon state defined by $2 \chi=\pi / 10$ and $2 \varphi=0$ (see Fig. 4). In order to reconcile both behaviors, principally because no trajectory should be expected at a nodal region (these are regions of lowest probability), it is important to observe the time dependence of $x_{1}$ and $x_{2}$, displayed in Figs. 4(c) and 4(d), respectively. In the case of $x_{1}$, we note a bistable behavior. That is, $x_{1}$ is essentially +1 or -1 for relatively long periods of time (which are expected to increase as $2 \chi \rightarrow 0$ ), while $x_{2}$ only oscillates linearly, up and down, between these values to compensate the transition from $x_{1}$ to $x_{2}$. We observe that such transitions are extremely fast. Actually, in the case of $x_{1}$, the velocity $v_{1}$ is negligible except at the transition times, while $v_{2}$ fluctuates between two almost stationary values $(+0.16$ and -0.16 , approximately). As a consequence, the system will remain apparently either in one lobe or the other of $P\left(x_{1}, x_{2}\right)$, as shown by the orbit of the velocity representation displayed in Fig. 4(b) (if we consider $2 \varphi \rightarrow \pi$, we will find the same, but replacing $x_{1}$ by $x_{2}$, and vice versa).

The situation is similar if we move around the equatorial plane regarding the presence of the two lobes in $P\left(x_{1}, x_{2}\right)$, although this motion implies a rotation of the axis along which they are distributed. As mentioned in Sec. IIC, the lobes are aligned with the $x_{1}$ axis for $2 \varphi=0$ and start inclining counterclockwise as this angle increases, becoming aligned with the $x_{2}$ axis when $2 \varphi=\pi$. This change affects not only the distribution $P\left(x_{1}, x_{2}\right)$, but also the phase of the single-photon state and therefore its associated trajectory dynamics. To illustrate this case, we are going to consider the state described by $2 \chi=\pi / 10$ and $2 \varphi=\pi / 2$ (see Fig. 5 ), which is linked to the distribution displayed in the lowest right panel of Fig. 2. Although the trajectory is still circular, the orbit in the velocity space describes two lobes distributed along a $\pi / 4$ axis [see Fig. 5(b)], which in the limit $2 \chi \rightarrow 0$ will end up in a line with a $\pi / 4$ inclination. Note that these lobes constitute a distortion of those displayed by the velocities in the previous case [compared with Fig. 4(b)]. In Figs. 5(c) and 5(d) we observe that this behavior just means that the photon oscillates between one lobe of the distribution $P\left(x_{1}, x_{2}\right)$ and the other in a rather inhomogeneous way: there is a slow, gradual approach to the transition followed by a sudden switch, as indicated by the corresponding velocities. In the corresponding phase spaces [see Figs. 5(e) and 5(f)], we observe precisely that the velocity reaches its maximum value right after the photon has reached one of the lobes [of $P\left(x_{1}, x_{2}\right)$ ] and just before it jumps into the other. Nonetheless, although the angular velocity is rather nonuniform, the trajectory is still circular.

Finally, we would like to illustrate the source of motion in the kind of stationary state considered here. In contrast to
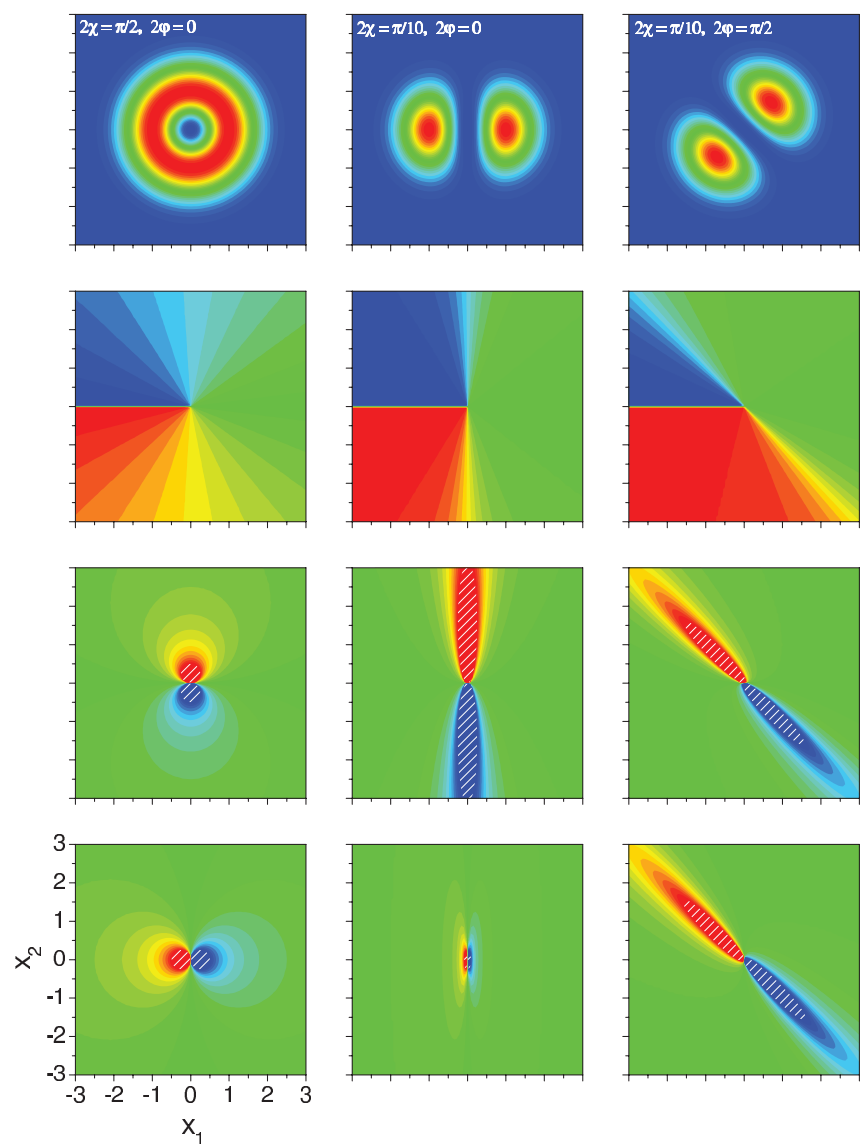

FIG. 6. (Color online) Distribution $P\left(x_{1}, x_{2}\right)$ (top row), phase field $S$ (second row), and components of the local velocity field, $v_{1}$ and $v_{2}$ (third and fourth rows, respectively), for the polarization states considered in Figs. 3, 4, and 5 (left, central, and right columns, respectively). Minimum to maximum is indicated with the transition from blue to red: for $P\left(x_{1}, x_{2}\right)$, starting from zero in all cases; for $S$, between $-\pi$ and $+\pi$; and for $v_{1}$ and $v_{2}$, between -2 and +2 . In the last two rows the shaded areas indicate a truncation to the maximum and minimum values chosen.

nonstationary wave functions, where the time dependence of the phase leads naturally to time-evolving trajectories [13,14], here motion has a topological origin: the space-dependent (configuration space) gradient of the global phase associated with the stationary state. In Fig. 6 we show the distribution $P\left(x_{1}, x_{2}\right)$ (upper row), the phase $S$ (second row), and the components of the local velocity field, $v_{1}$ and $v_{2}$ (in the third and fourth rows, respectively), of the three cases represented in Figs. 3, 4, and 5 (left, central, and right columns, respectively). From a quick inspection, the role of the phase-field topology on the trajectory dynamics becomes readily apparent. This field decreases monotonically counterclockwise, thus explaining the evolution of the trajectories (from their beginning, as $x_{1}$ decreases, $x_{2}$ increases). Now, while this decrease is gradual for $2 \chi=\pi / 2$, it becomes steplike as $2 \chi$ approaches zero. In the latter case, the step lies precisely along the symmetry axis passing between the two lobes. In this sense, the motion is relatively slow along each plateau of the phase field and very fast when the trajectory comes down the step (i.e., passes from the region covered by one of the lobes to the region covered by the other). This has a straightforward counterpart in the 
corresponding velocity field components. For $2 \chi=0$ these components are symmetric with respect to a $\pi / 2$ rotation, which is a signature of harmonic motion (as one of the components starts to decrease, the other increases, and vice versa). Now, as $2 \chi$ approaches zero we find two types of behaviors. For $2 \chi=0$ and $2 \varphi=0$, the component along the step of the phase field becomes more prominent than the component that is perpendicular to this step, which eventually becomes meaningless. This is in correspondence with the fact that it is more likely to find the trajectory either in the right or the left lobe of $P\left(x_{1}, x_{2}\right)$ (small $v_{1}$ and $v_{2}$ ), but not in between (large $v_{1}$ ). A similar behavior, but exchanging the axes, would be found for $2 \chi=0$ with $2 \varphi=\pi$. Now, as $2 \varphi$ moves towards $\pi / 2$, the two components tend to align, thus becoming equal for $2 \varphi=\pi / 2$, which means that the trajectories will avoid staying in the neighborhood of the diagonal $x_{2}=-x_{1}$.

\section{CONCLUSIONS}

In this work we have shown that the topology displayed by the Bohmian trajectories analyzed has no relation whatsoever with the form of the polarization ellipse of the corresponding field state, since they are always circles. However, the angular speed at which these circles are described contains complete information about the mean polarization state, i.e., the ellipse traditionally associated with the Stokes parameters. This information is paradoxically absent from the probability distribution corresponding to the $2 \mathrm{D}$ real electric field [see Eq. (12)], where one would expect to see the quantum counterpart of the classical mean ellipse. Moreover, seemingly, the Bohmian trajectories provide no hint about the uncertainty of the polarization state, since the families of trajectories associated with a given field state are essentially identical.

Concerning the dynamics associated with single-photon polarization states, we have found a relatively rich variety of behaviors depending on the point on the Poincaré sphere defining the state vector. As seen, although all the trajectories are circles, their dynamical evolution is strongly dependent on their position with respect to the Poincaré sphere, ranging thus from harmonic behaviors (for trajectories on the poles) to bistable oscillating ones (for trajectories on the equator). In all cases, though, this dynamics has a clear topological origin associated with the particular shape of the phase field, which goes from a gradual decrease around $\left(x_{1}, x_{2}\right)=(0,0)$ (at the poles) to a steplike one (on the equator). Accordingly, trajectories evolve counterclockwise either harmonically or displaying jumps, respectively.

The fact that polarization stationary states may have an associated internal or intrinsic dynamics is puzzling as well as challenging, for it goes beyond our physical intuition even if it is proven mathematically, as was done here. Even though distributions like $P\left(x_{1}, x_{2}\right)$ are invariant objects with time, there is a dynamics induced by the phase-field topology. This poses the interesting and stimulating question of whether such an intrinsic motion could be experimentally measured. Note that recently Steinberg and co-workers were able to experimentally infer [15] the Bohmian trajectories or averaged photon paths [7] in a two-slit experiment by means of weak measurements $[16,17]$. The ideas posed here clearly point towards a further extension of this work in this direction with the ultimate goal of performing such experimental measurements. This would allow us to better understand the nature of stationary states as situations where the system is statistically described by a time-independent (steady) distribution, but that display particular inner dynamics. Obviously, this has a potential interest in quantum information and quantum computation, where the basic ingredient, the qubit, is precisely described by a state like (9). Furthermore, also notice the implication in quantum mechanics, where the role equivalent to polarization is played by the particle spin. This vectorial quantity is traditionally assigned to an internal rotation, because it fulfils the same properties of the rotation group. With the picture on stationary states provided above, based on dynamical grounds, this connection becomes even clearer.

\section{ACKNOWLEDGMENTS}

Support from the Ministerio de Economía y Competitividad (Spain) under Projects No. FIS2012-35583 (A.L.), No. FIS2010-22082 (A.S.), and No. FIS2011-29596-C0201 (A.S.), and a "Ramón y Cajal" Grant (A.S.); from the Consejería de Educación de la Comunidad de Madrid under Project No. QUITEMAD S2009-ESP-1594 (A.L.); and from the COST Action MP1006 "Fundamental Problems in Quantum Physics" (A.S.) is acknowledged. A.S. also thanks the University College London for its kind hospitality.
[1] M. Born and E. Wolf, Principles of Optics, 7th expanded ed. (Cambridge University Press, Cambridge, England, 1999); Ch. Brosseau, Fundamentals of Polarized Light: A Statistical Optics Approach (Wiley, New York, 1998).

[2] J. Schwinger, Quantum Theory of Angular Momentum (Academic Press, New York, 1965).

[3] J. Pollet, O. Méplan, and C. Gignoux, J. Phys. A 28, 7287 (1995).

[4] A. Luis, Phys. Rev. A 66, 013806 (2002).

[5] J. Liñares, M. C. Nistal, D. Barral, and V. Moreno, Eur. J. Phys. 31, 991 (2010); J. Liñares, D. Barral, M. C. Nistal, and V. Moreno, J. Mod. Opt. 58, 711 (2011).

[6] D. Bohm, Phys. Rev. 85, 166 (1952); 85, 180 (1952); P. R. Holland, The Quantum Theory of Motion (Cambridge University
Press, Cambridge, England, 1993); A. S. Sanz and S. MiretArtes, Am. J. Phys. 80, 525 (2012); X. Oriols and J. Mompart, in Applied Bohmian Mechanics: From Nanoscale Systems to Cosmology, edited by X. Oriols and J. Mompart (Pan Stanford Publishing, Singapore, 2012), Chap. 1, pp. 15-147.

[7] A. S. Sanz, M. Davidović, M. Božić, and S. Miret-Artés, Ann. Phys. (NY) 325, 763 (2010).

[8] A. S. Sanz, J. Campos-Martínez, and S. Miret-Artés, J. Opt. Soc. Am. A 29, 695 (2012).

[9] R. Delbourgo, J. Phys. A 10, 1837 (1977).

[10] A. Picozzi, Opt. Lett. 29, 1653 (2004); Ph. Réfrégier, ibid. 30, 1090 (2005); A. B. Klimov, L. L. Sánchez-Soto, E. C. Yustas, J. Söderholm, and G. Björk, Phys. Rev. A 72, 033813 (2005); Ph. 
Réfrégier and F. Goudail, J. Opt. Soc. Am A 23, 671 (2006); L. L. Sánchez-Soto, J. Söderholm, E. C. Yustas, A. B. Klimov, and G. Björk, J. Phys.: Conf. Ser. 36, 177 (2006); I. Ghiu, G. Björk, P. Marian, and T. A. Marian, Phys. Rev. A 82, 023803 (2010).

[11] F. T. Arecchi, E. Courtens, R. Gilmore, and H. Thomas, Phys. Rev. A 6, 2211 (1972).

[12] O. Giraud, P. Braun, and D. Braun, Phys. Rev. A 78, 042112 (2008).
[13] A. S. Sanz and S. Miret-Artes, Chem. Phys. Lett. 445, 350 (2007).

[14] A. S. Sanz and S. Miret-Artes, J. Phys. A 41, 435303 (2008).

[15] S. Kocsis, B. Braverman, S. Ravets, M. J. Stevens, R. P. Mirin, L. K. Shalm, and A. M. Steinberg, Science 332, 1170 (2011).

[16] Y. Aharonov and L. Vaidman, Phys. Rev. A 41, 11 (1990).

[17] B. Hiley, J. Phys.: Conf. Ser. 361, 012014 (2012). 